\title{
Development of a Tomographic System for Online Dose Measurements in BNCT (Boron Neutron Capture Therapy)
}

\author{
A. Valda, D. M. Minsky, A. J. Kreiner,* A. A. Burlon, and H. Somacal \\ Escuela de Ciencia y Tecnología (UNSAM), San Martín, Buenos Aires, Argentina and \\ Dpto. de Física, Centro Atómico Constituyentes, Comisión Nacional de Energía Atómica, Buenos Aires, Argentina
}

Received 19 July, 2005

\begin{abstract}
Within our activities on accelerator-based boron neutron capture therapy (BNCT) carried out at the Tandar Laboratory (Comisión Nacional de Energía Atómica, Argentina) we present here the study and design of a tomographic imaging system for the measurement of the spatial distribution of the absorbed dose during a BNCT session. The ${ }^{10} \mathrm{~B}(n, \alpha){ }^{7} \mathrm{Li}$ boron neutron capture reaction produces a $478 \mathrm{keV}$ gamma ray in $94 \%$ of the cases. In BNCT a large fraction of this radiation escapes from the patient body. Its detection is thus very attractive for a noninvasive boron concentration measurement and an online absorbed dose evaluation since the dose absorbed by the tumor and healthy tissue strongly depends on the boron uptake and the neutron flux. For this purpose, a dedicated tomographic imaging system based on SPECT (Single Photon Emission Computed Tomography, a diagnostic medical imaging modality used in nuclear medicine) is proposed since standard SPECT cameras cannot be used due to the photon energy $(478 \mathrm{keV})$ and the particular background gamma field in BNCT. A detection system (collimator and detectors) is proposed. Monte Carlo numerical simulations are used for an implementation of a statistical algorithm used for the tomographic image reconstruction.
\end{abstract}

\section{INTRODUCTION}

Boron neutron capture therapy (BNCT) [1] is a radiation therapy under development for the treatment of some cancers like melanoma and glioblastoma multiforme. It is performed in two steps: first, a stable isotope of boron $\left({ }^{10} \mathrm{~B}\right)$ is administered to the patient via a carrier drug and then the patient is irradiated with an epithermal neutron beam. ${ }^{10} \mathrm{~B}$ will then undergo the capture reaction $\left.{ }^{10} \mathrm{~B}(n, \alpha)\right)^{7} \mathrm{Li}\left({ }^{10} \mathrm{~B}\right.$ capture cross section for thermal neutrons: $3840 \mathrm{~b}$ ). The emitted charged particles $(\sim 1 \mathrm{MeV})$ have a high linear energy transfer (LET) and are lethal only to the cells in close proximity to the reaction point because their range is $\sim 10 \mu \mathrm{m}$. Reactor-based epithermal neutron beams have been developed to treat patients (e. g. [2] - [4]). As an alternative to reactors, the application of accelerator-based neutron sources for BNCT (AB$\mathrm{BNCT}$ ) is being proposed at a number of laboratories (e. g. [5] - [8]). Among other advantages over reactor sources, accelerators can produce more appropriate neutron spectra with low fast neutron and gamma ray contamination of the therapy beam.

The dosimetric aspects of BNCT are very complex due to the several interactions of neutrons with the different nuclei appearing in healthy and tumor tissue. Nevertheless the absorbed dose in the tumor is dominated by the ${ }^{10} \mathrm{~B}$ capture reaction. Usually the evolution of the ${ }^{10} \mathrm{~B}$ concentration in tumor and in healthy tissue is obtained indirectly from blood samples taken from the patient before, after and during the BNCT treatment since tumor-to-blood and healthy tissue-to-blood ${ }^{10} \mathrm{~B}$ concentrations are inferred from previous biodistribution studies of the ${ }^{10} \mathrm{~B}$ carrier. Hence a better measurement of ${ }^{10} \mathrm{~B}$ concentration during a BNCT treatment will result in a better

*Also CONICET, Argentina knowledge of the dose received by the patient and its relation to the treatment response. The external detection of the $478 \mathrm{keV}$ prompt-gamma radiation (linear attenuation coefficient for soft tissue is $\sim 0.1 \mathrm{~cm}^{-1}$ ) with spatial discrimination should serve as the basis for an online dose measurement in a noninvasive way. In this sense, a device called gamma-ray telescope has been developed and is in use at the BNCT facility in Petten, the Netherlands [8]. In Japan, Kobayashi et al. [9] have presented a feasibility study of a tomographic system based on single photon emission tomography (SPECT). In this work we propose a tomographic system, also using the SPECT principle, based on pixelated scintillator detectors. In the first section we evaluate the prompt-gamma production issued from the capture in ${ }^{10} \mathrm{~B}$ based on expected results of $\mathrm{AB}$ $\mathrm{BNCT}$ applied to brain tumors. The next section is devoted to the description of the collimator and radiation detector of the proposed system. Finally we assess the imaging performance of the system by Monte Carlo numerical simulations using a simple phantom as radiation source. An iterative statistical algorithm (Expectation- Maximization Maximum-Likelihood) is used for the single slice reconstruction.

\section{ASSESSMENT OF $478 \mathrm{keV}$ PROMPT-GAMMA PRODUCTION IN BNCT}

In BNCT the dose delivered to the tumor is usually limited by the maximum dose that the healthy tissue can receive which is dependent on its boron concentration (nonspecific drug uptake) and on the neutron beam quality. From our previous work [10] on accelerator-based BNCT applied to brain tumors and using $p$-boronphenylalanine (BPA) as the boronated compound, it is possible to estimate a tumor dose of 40 RBE-Gy in a BNCT treatment. The ${ }^{10} \mathrm{~B}$ capture reaction dose component accounts for about $80 \%$ of this value. Considering a mean energy per capture reaction of $2.31 \mathrm{MeV}$, one obtains of the order of $2 \cdot 10^{9}$ emissions of $478 \mathrm{keV}$ photons 
per cubic centimeter of tumor. This is the maximum number of photons available for the online dose measurement; owing to the attenuation in soft tissue, this number should be corrected by a factor of about 0.37 . Concentration measurements require the use of tomographic approaches, the most appropriate for this application is that based on the nuclear medicine image modality known as SPECT, for Single Photon Emission Tomography [11].

\section{SYSTEM DESIGN}

In SPECT, a bidimensional position sensitive detector (the gamma camera) detects the gamma rays emitted from the patient. The gamma camera is composed of the collimator, the radiation detector itself and a coding electronics. The collimator selects the direction of incidence of the gamma rays into the detector. It consists of a large number of holes in an absorbing material like lead. The direction of the holes determines the direction of the accepted radiation (usually perpendicular to the detection plane). The geometrical characteristics of the collimator mostly determine the imaging properties of the system (spatial resolution and sensitivity). In clinical SPECT the detector is a continuous $\mathrm{NaI}(\mathrm{Tl})$ crystal optically coupled to a set of photomultiplier tubes (PMT). The relative signal amplitudes from the PMTs is electronically analyzed (Anger logic) in order to give the spatial position of the impact of the gamma ray on the detector.

\section{A. Collimator}

The round-hole parallel collimator in lead was designed on the basis of geometrical calculations and estimates of the photon attenuation in collimator material. The expression used for the geometrical spatial resolution $\left(R_{g}\right)$ was

$$
R_{g}=\frac{\phi(e+d)}{e}
$$

where $\phi$ is the collimator diameter, $e$ the collimator thickness (length of holes) and $d$ the distance from the point where $R_{g}$ is calculated to the collimators entrance face. The septum thickness or thickness of material between adjacent holes $(t)$ was calculated by numerically solving the equation:

$$
\frac{t}{l}=\frac{2 \phi+t}{\sqrt{e^{2}+(2 \phi+t)^{2}}}
$$

where $l$ is the length of lead that attenuates a given amount of $478 \mathrm{keV}$ photons (linear attenuation coefficient $1.97 \mathrm{~cm}^{-1}$ ). The geometrical point source sensitivity $\left(\varepsilon_{g}\right)$ was obtained by evaluating the solid angle subtended by all hole apertures, which depends on $\phi, e, d$ and $t$ (since $\phi+t$ is the center-tocenter hole separation). The collimator geometry is then obtained by maximizing $\varepsilon_{g}$ for a given spatial resolution $R_{g}$, at a given distance $d$ and allowing a given septal attenuation.
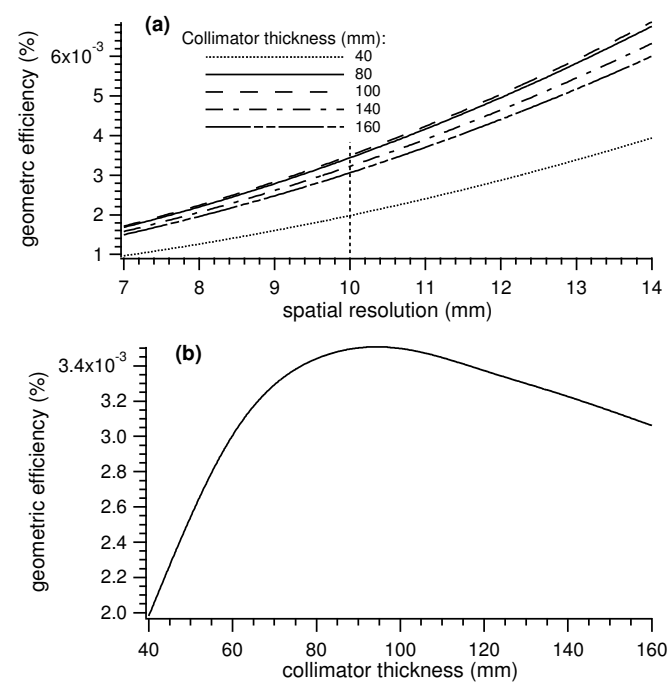

FIG. 1: (a) Collimator geometric efficiency as a function of spatial resolution for different collimator thicknesses. Distance sourcecollimator: $20 \mathrm{~cm}$, septal attenuation: $94 \%$. The vertical dotted line intersects the curves at $1 \mathrm{~cm}$ of spatial resolution. (b) Geometric efficiency as a function of collimator thickness for $1 \mathrm{~cm}$ of spatial resolution (other parameters as in (a))

For this application we chose $l=1.54 \mathrm{~cm}$ (which provides an attenuation of 94\%). Figure 1.a shows a family of efficiency vs. resolution curves for several collimator thicknesses; the resolution is taken at $d=20 \mathrm{~cm}$. Considering $R_{g}=1 \mathrm{~cm}$ at $20 \mathrm{~cm}$ from the collimator, it is possible to see that the efficiency is maximum $\left(\varepsilon_{g}=3.5 \cdot 10^{-3} \%\right)$ for $e=9.4 \mathrm{~cm}$. Figure 1.b presents a complementary visualization of this result. However, with the aim of diminishing the weight of the collimator structure we have chosen to work in a suboptimal situation with $e=8 \mathrm{~cm}$ that gives $\varepsilon_{g}=3.4 \cdot 10^{-3} \%$ (2\% less than the optimum). In order to largely cover a transaxial fieldof-view of $20 \mathrm{~cm}$, a collimator of $23.7 \mathrm{~cm}$ in lateral length was chosen; such a collimator has 37 parallel holes.

\section{B. Detectors}

In previous work [12] on the characterization of a neutron beam obtained from an accelerator, we have measured with a high-purity germanium (HPGe) detector typical gamma ray spectra during the irradiation of a cylindrical head phantom containing a small $(1.6 \mathrm{~cm}$ in diameter, $2 \mathrm{~cm}$ in height) cylinder with boron (Fig. 2). The choice of the radiation detector is related to the presence of the $511 \mathrm{keV}$ annihilation peak near the $478 \mathrm{keV}$ prompt gamma peak. The annihilation peak is originated as a consequence of electron-positron pair creation after interaction of high energy gamma rays, mostly $2.23 \mathrm{MeV}$ issued from neutron capture in hydrogen. In order to avoid the use of a cooling system (needed for HPGe), our attention was focused on a CdZnTe detector (a semiconductor detector which operates at room temperature) and on a $\mathrm{LaCl}_{3}(\mathrm{Ce})$ scintillator. $\mathrm{CdZnTe}$ has an appropriate energy re- 


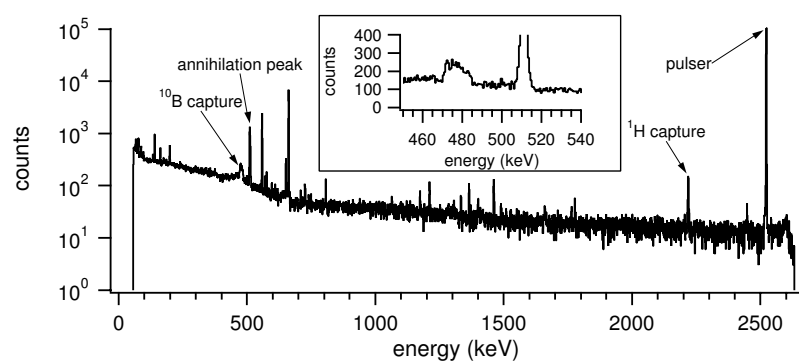

FIG. 2: HPGe spectrum obtained during the irradiation of a cylindrical head phantom containing a small cylinder with boron. Relevant peaks are indicated. The inset is a closer view of the region around the 478 and $511 \mathrm{keV}$ peaks. The broadening of the ${ }^{10} \mathrm{~B}$ capture peak is caused by the Doppler effect since the prompt gamma is emitted "in flight" by the ${ }^{7} \mathrm{Li}$ ion.

solution of $1.8 \%$ at $662 \mathrm{keV}(\mathrm{FWHM}=12 \mathrm{keV})$ but the present technology limits the fabrication of these crystals to about $5 \mathrm{~mm}$ in thickness, which provides an intrinsic efficiency of only $4 \%$. On the other hand, the recently developed $\mathrm{LaCl}_{3}(\mathrm{Ce})$ crystal, a high light yield scintillator ( 49 photons $/ \mathrm{keV})$, can be constructed with a more appropriate thickness. For example $2.5 \mathrm{~cm}$ of $\mathrm{LaCl}_{3}(\mathrm{Ce})$ provide an intrinsic efficiency of about $16 \%$. Even if $\mathrm{LaCl}_{3}(\mathrm{Ce})$ has worse energy resolution than CdZnTe, it is however good enough for our application $(\mathrm{FWHM}=28 \mathrm{keV}$ for $662 \mathrm{keV})$ [13].

In order to have appropriate statistics for the tomographic reconstruction during the treatment time it is necessary to surround the patient with a sufficient number of detector arrays, which will provide the projection data. Considering the total number of prompt gamma events produced in $1 \mathrm{~cm}^{3}$ tumor (cf. II) and 5 arrays of $\mathrm{LaCl}_{3}(\mathrm{Ce})$ detectors coupled to the collimator described above (each having a detection efficiency of $2 \cdot 10^{-6}$ ), a total of $2 \cdot 10^{4}$ photons $/ \mathrm{cm}^{3}$ of $478 \mathrm{keV}$ could be detected. This array should be successively positioned at 4 different angles around the patient, as we will see in the next section, giving a total of 1000 photons per position and per array issued from a tumor of $1 \mathrm{~cm}^{3}$ during the whole treatment time. Therefore, if one wants to monitor the treatment at its mid-time point, it should be possible to have a statistical fluctuation less than $10 \%$ in the determination of the prompt gamma production in a tumor of $1 \mathrm{~cm}^{3}$.

\section{NUMERICAL SIMULATIONS AND TOMOGRAPHIC RECONSTRUCTION}

\section{A. Acquisition data}

The simulations were performed using the Monte Carlo simulation code MCNP-4c [14]. Two phantom sources were used to simulate the acquired (projection) data: (i) a set of four parallel "hot" cylinders with diameters $0.5,1,1.5$ and $2 \mathrm{~cm}$ and (ii) the same set immersed in a $20 \mathrm{~cm}$ diameter cylinder for simulating a background source with a size comparable to a human head. In the last case the ${ }^{10} \mathrm{~B}$ concentration a)

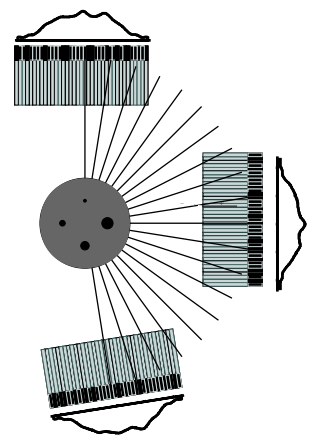

b)
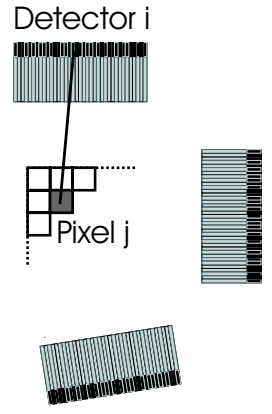

FIG. 3: Geometric configurations considered in the Monte Carlo simulations. (a) Simulation of the projection data of the phantoms: there are 37 detectors in each of the 20 angular positions of the array. (b) Calculation of the element $i j$ of the system matrix.

in the four small cylinders was the same and the cylindersto-background concentration ratio was $4: 1$, like in a typical BNCT case. One-dimensional projections were taken around the cylinders in order to reconstruct bi-dimensional transaxial planes (the three-dimensional volume can be reconstructed by stacking independent bi-dimensional planes). Each angular position of a detector plane is composed of 37 line projections according to the collimator-detector described in the previous section. A total of 20 angular positions (evenly spaced at 9 degrees) were considered (Figure 3.a) giving a total of 740 projection data. The number of simulated events is such that the statistical fluctuations of the counting process can be neglected.

\section{B. Image reconstruction}

Considering the small number of available angular positions, analytical tomographic reconstruction algorithms were discarded. Instead we have chosen a statistical iterative algorithm, the so-called Expectation Maximization - Maximum Likelihood (EM-ML) algorithm [15]. In order to model the system matrix $\mathbf{A}$, taking into account attenuation and scatter in collimator and photoelectric absorption in the scintillator, we calculate the matrix elements $a_{i j}$ by Monte Carlo simulations using the MCNP-4c code (Figure 3.b). For this process we consider a reconstruction matrix size of $21 \times 21$ voxels (voxel size $1 \mathrm{~cm} \times 1 \mathrm{~cm} \times 1 \mathrm{~cm}$ ). The projections are obtained under the same conditions described above for the acquisition data. The resulting system matrix size has then $441 \times 740$ elements. The reconstructed phantom projection data are shown in figure 4 for the sources without (100 iterations) and with background (225 iterations). All the sources can be observed in both cases. As expected from partial volume effects, the best signal-to-background relation is obtained for the $2 \mathrm{~cm}$ cylinder (namely 4:1); for the $1 \mathrm{~cm}$ cylinder this ratio is around 3:1. 

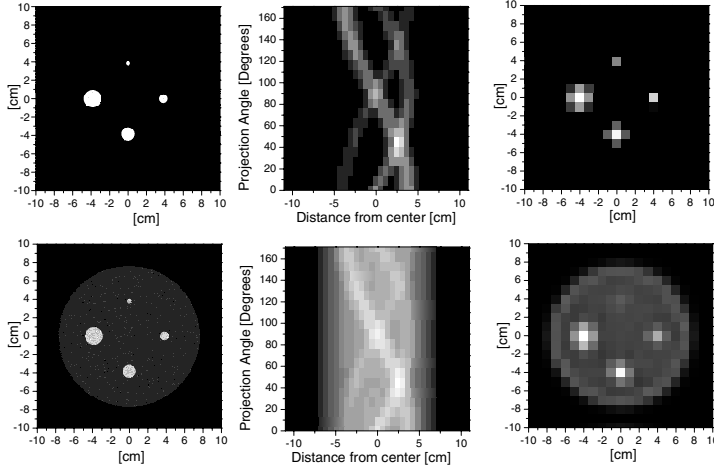

FIG. 4: Simulated reconstructed images of the hot-rod phantoms: without background (upper row) and with background (4:1) (lower row). From left to right: original phantom, sinogram and reconstructed image.

\section{CONCLUSIONS}

A tomographic device for spatially resolved online dosimetry in BNCT has been proposed. Monte Carlo numerical si- mulations are used to evaluate its imaging performance. First results indicate that it would be possible to recover boron concentrations in tumors with $2 \mathrm{~cm}$ in diameter. As the accuracy of this determination depends on the counting statistical fluctuations, future numerical work points in this direction. We are also working in the correction for in-phantom photon attenuation. Anthropomorphic phantoms will be used to explore the system performance better.

\section{Acknowledgments}

The authors wish to thank the TANDAR personnel for their collaboration. Special thanks are addressed to the END group (CNEA Physics Department) members, M. E. Caraballo, M. Debray, M. Davidson, J. Davidson, J. M. Kesque, M. Ozafrán, and M. E. Vázquez for their contribution to the experimental aspects of this work. We gratefully acknowledge the support provided by the National Agency for the Promotion of Science and Technology, ANPCyT.
[1] J. A. Coderre and G. M. Morris, Radiat. Res. 151, 1 (1999) and references therein.

[2] R. Rogus, O. Harling, and J. Yanch, Med. Phys. 21, 1611 (1994)

[3] C. Vroegindeweij, P. Watkins, and R. L. Moss; Frontiers in neutron capture therapy; M. F. Hawthorne, K. Shelly and R. J. Wiersema, eds. (New York: Kluwer Academic/Plenum Publishers), 375 (2001).

[4] H. R. Blaumann, S. J. González, J. Longhino et al., Med. Phys. 31, 70 (2004).

[5] R. Klinkowstein, R. Schefer, J.C. Yanch, et al., Proceedings of the 7th International Symposium on Neutron Capture Therapy for Cancer, Vol. 1; B. Larsson, J. Crawford and R. Weinreich, eds.; (Amsterdam, Elsevier Science B. V.), 522 (1997).

[6] A. A. Burlon, A. J. Kreiner and A.Valda; Proceedings of the Sixth Mexican Symposium; L. M. Montano Zetina and G. Herrera Corral, eds. (AIP Conference Proceedings), 54 (2002).

[7] C. N. Culbertson, S. Green, A. J. Mason et al., Applied Radiation and Isotopes 61, 733 (2004).

[8] W. F. A. R. Verbakel, W. Sauerwein, K. Hideghety, and F.
Stecher-Rasmussen, Int. J. Radiation Oncology Biol. Phys. 55, 743 (2003).

[9] T. Kobayashi, Y. Sakurai, and M. Ishikawa, Med. Phys. 27, 2124 (2000).

[10] A. A. Burlon, A. J. Kreiner, A. A. Valda, and D. M. Minsky, Applied Radiation and Isotopes 61, 811 (2004).

[11] See for example P. Suetens, "Fundamentals of medical imaging"; Cambridge University Press, 2002.

[12] A. A. Burlon, A. J. Kreiner, A. A. Valda et al., Nucl. Instr. and Meth. B 229, 144 (2005).

[13] E. V. D. van Loef, W. Mengesha, J. D. Valentine et al., IEEE Trans. Nucl. Sci. 50, 155 (2003).

[14] J. F. Briesmeister, "A general Monte Carlo n- particle transport code", Los Alamos, NM, Los Alamos National Laboratory, (2000).

[15] L. A. Shepp and Y. Vardi, IEEE Trans. Med. Imaging MI-1, 113 (1982). 